\title{
El lugar del sujeto en el campo de la salud: enseñanzas de la investigación cualitativa
}

\author{
The place of the individual in the field of health: \\ lessons from qualitative investigation
}

${ }^{1}$ Departamento de Atención a la Salud, Universidad Autónoma Metropolitana (campus Xochimilco).

Calz del Hueso 1100 Villa Quietud, Coyoacán. 04960 Ciudad de México Distrito Federal México.

cmartine@correo.xoc.uam.mx
Abstract This article addresses some questions regarding the exclusion of the subjectivity derived from the ideals of objectivity and neutrality that characterize the modern scientific parade gm. Several consequences of this exclusion with respect to health care and illness are considered. Five problem areas are discussed, emphasizing the contributions of qualitative inquiry for the recovery of the importance of the individual. These include health and disease as subjective experiences, the gap between the experts' perspective and that of the layman, as well as disagreements among health professionals and common people, both in the preventive and in the curative fields. They also include the omission of the patients' voice when dealing with their illness and the sterilization of subjective creativity to establish new avenues to health care. The paper concludes by pointing out that, despite the challenges involved, certain currents of qualitative research whose foundations are located in the most advanced area of current epistemological, ethical and aesthetic reflection, can help to open new routes towards richer alternatives for health care and illness in Latin American societies.

Key words Qualitative investigation, Critical perspective, Health care, Subjectivity
Resumen En este artículo se cuestiona la exclusión de la subjetividad derivada de los ideales de objetividad y neutralidad del paradigma científico moderno. Se exponen algunas de sus consecuencias en lo que atañe a la construcción de la salud y el cuidado de la enfermedad. Se discuten cinco nudos problemáticos en los que la investigación cualitativa tiene valiosos aportes qué ofrecer para la recuperación del lugar del sujeto: la salud y la enfermedad como experiencias subjetivamente percibidas y significadas; la brecha que separa a la visión de los expertos y de quienes no lo son; los desencuentros entre los profesionales de la salud y la población, tanto en el frente curativo como en el preventivo; el silenciamiento de la voz del enfermo sobre su vivencia de la enfermedad; $y$ la esterilización de la creatividad subjetiva en la construcción de rutas más diversas para el cuidado de la salud. Se postula que, con todos los retos implicados, ciertas corrientes de la investigación cualitativa cuyos fundamentos se ubican en el polo más avanzado de la reflexión epistemológica, ética e incluso estética de nuestros días, pueden ayudar a abrir nuevas rutas hacia alternativas más ricas e imaginativas para el cuidado de la saludy la atención de la enfermedad en las sociedades latinoamericanas.

Palabras-clave Investigación cualitativa, Perspectiva crítica, Atención a la salud, Subjetividad 


\section{Introducción}

Las ciencias de la salud, con un pie en el terreno de las ciencias naturales y otro en el de las ciencias sociales, ocupan una posición a la vez privilegiada y extremadamente conflictiva, tanto en el mundo del conocimiento como en el de la práctica. De la tradición científico natural, los profesionales de la salud hemos heredado los ideales de neutralidad y objetividad, fundados en un paradigma que ha permitido importantes avances en el conocimiento sobre la forma en la que está constituido y funciona el organismo humano. Pero del pensamiento científico social hemos tenido que aprender que la ciencia no es "un reflejo fiel y exacto de la realidad", sino un complejo entramado interpretativo sobre el mundo, resultado de la creación humana, cuyos postulados no vienen de otro lado que no sea la actividad intersubjetiva creadora de sentido que ha sedimentado a lo largo de los siglos ${ }^{1}$.

De esta suerte, aún cuando el paradigma científico moderno opere bajo la pretensión de excluir la subjetividad para construir un conocimiento objetivo y neutral, el sujeto no puede desaparecer de la escena, en tanto que es él quien concibe, observa, piensa, interpreta los fenómenos en estudio, es decir, el que otorga su sentido óntico al mundo ${ }^{1,2}$. Y, como nos lo hacen ver los pensadores que cultivan perspectivas críticas, el sujeto nunca es neutral; el intérprete habla siempre desde una determinada posición ${ }^{3,4}$.

Por otra parte, la experiencia cotidiana nos enfrenta con la evidencia de que nuestra "materia de trabajo" no está constituida en modo alguno por objetos inertes, dóciles a nuestras indicaciones o a nuestra manipulación ${ }^{5-7}$. Una cosa son los tejidos, células y moléculas que estudiamos en el laboratorio, y otra las personas que viven en el mundo y se acercan en busca de ayuda a los servicios médicos y sanitarios. Cuando esto último se deja de lado, el sofisticado conocimiento logrado por las ciencias biomédicas resulta desproporcionadamente simplista e ingenuo.

Pensando en las sociedades latinoamericanas, constituidas por poblaciones tan extremadamente diversas no sólo en lo que se refiere a sus condiciones materiales de vida sino también a sus mentalidades, en las siguientes páginas quiero ocuparme de cinco nudos problemáticos en los cuales la investigación cualitativa ${ }^{8,9}$ nos ayuda a entender y a asumir la imposibilidad de excluir al sujeto en lo que se refiere al estudio de la salud y su cuidado.

\section{Primer nudo problemático: la salud y la enfermedad como experiencias subjetivas}

Los aportes del conocimiento científico natural, incluso los más recientes avances de la biología molecular, por valiosos que resulten para descifrar el modo de funcionamiento del organismo humano, no bastan para entender los complejos procesos involucrados en la construcción de la salud y la ocurrencia de la enfermedad. Porque lo que queda todavía por considerar es cómo se constituye la corporeidad en el ámbito intersubjetivo en el que el sujeto nace, en medio de un conjunto de relaciones sociales inscritas en una totalidad histórica ${ }^{10,11}$. En efecto, las personas no son un conjunto de tejidos, órganos y funciones estructuradas a través de mecanismos exclusivamente biológicos; las dimensiones simbólica, afectiva y volitiva no son secreciones de los aparatos y sistemas del organismo humano, que está muy lejos de ser una masa biológica muda. La materialidad biológica humana es una realidad diferente de la que se observa en otros niveles de organización de la materia viva; es una realidad que se elabora simbólicamente, en la que se sintetizan las vertientes que la constituyen - la biológica y la social -, mediante complejas elaboraciones en el plano de lo psíquico ${ }^{12,13}$.

La práctica de la investigación cualitativa para el estudio de la experiencia de la enfermedad y la construcción de la salud se funda en el reconocimiento de esta complejidad del sujeto humano ${ }^{6,7,14,15}$. Nos proporciona evidencias de que no podemos circunscribirnos a las concepciones que reducen al humano a sus aspectos fisiológicos, patológicos y bioquímicos; ni siquiera a los cognitivos, como parecerían pretender quienes tienen la convicción de que de lo que se trata es de "educar", un concepto que suele reducirse al de la mera provisión de "información". Nos permite pensar en el sujeto como aquel que - se lo proponga conscientemente o no - va construyendo, cada día de su vida, su propia salud ${ }^{16}$; sufre - no sólo en su carne, sino también y simultáneamente en su fantasía, es decir, en su cuerpo simbolizado- cada momento de sus episodios de enfermedad, y es a partir de ello que toma los cursos de acción que le son imaginables, admisibles y acequibles.

\section{Segundo nudo problemático: la visión del experto y la del hombre común}

Como lo explican Berger y Luckman ${ }^{17}$, los humanos estructuramos nuestros modos de en- 
tender el mundo en los contextos sociales y culturales en que nacemos y vivimos, en los cuales impera un orden, unas creencias que constituyen la "realidad" socialmente establecida, un conjunto de conocimientos que compartimos con otros en las rutinas normales y auto-evidentes de la vida cotidiana. Para las personas comunes "la realidad" es ese mundo intersubjetivo del sentido común con relación al cual orientan su comportamiento subjetivamente significativo.

Pero a quien entra al campo de la ciencia moderna le ocurre algo adicional: como bien lo observa Berman ${ }^{18}$, entra a un mundo de abstracciones que violan las observaciones cotidianas: "Los alumnos que están formándose para tomar posiciones en esa cultura son rápidamente reeducados en el modo de percepción newtoniano/cartesiano/galileano". De ahí que las características del conocimiento científico y las del conocimiento de sentido común sean tan radicalmente distintas. Pese a ello, como también lo hace notar Berman ${ }^{18}$, ni siquiera los científicos escapan de ser las personas comunes que también son, de manera que: “(...) en forma privada y emocional, aún funcionamos en el mundo de sentido común de la experiencia inmediata (...). Incluso mantenemos trazas de animismo, a medida que pasan los años desarrollamos una relación casi personal con una lámpara o una silla preferida, a pesar de que 'sabemos' que no es más que madera o metal."

Pero los profesionales de la salud tendemos a comportarnos como si lo anterior no aconteciera. Nos sentimos autorizados a tomar al conocimiento científico como parámetro para evaluar la supuesta racionalidad del comportamiento cotidiano de "la gente". Tendemos a juzgar como irracional cualquier acción que no nos parezca compatible con los lineamientos que de él derivan, y a descalificar toda orientación que provenga del sentido común de las personas. Nos desentendemos de la distancia que separa el conocimiento de sentido común que orienta el comportamiento de la gente de los distintos grupos sociales en su vida cotidiana, y el conocimiento experto del que derivan las propuestas elaboradas en un terreno disciplinario tan cifrado y a la vez tan normativo como es el de las ciencias de la salud.

La investigación cualitativa nos ayuda a percatarnos de ello y, al asumirlo, nuestra forma de entender y atender la problemática de salud puede cambiar radicalmente ${ }^{15,19}$. Desde esta aproximación puede verse por qué no parece sensato pretender imputar el contexto de inteligibilidad del sofisticado saber médico de nuestros días, e incluso nuestras propias convicciones morales, a todos los integrantes del muy heterogéneo conjunto social ${ }^{6,7,20}$. La incorporación de miradas como la de la investigación cualitativa es, por eso, no sólo necesaria, sino urgente.

\section{Tercer nudo problemático: los desencuentros entre los profesionales de la salud y "la gente"}

Como lo ha estudiado la etnometodología ${ }^{21}$, las interacciones en la vida cotidiana se dan en contextos de mutua inteligibilidad en los que hay múltiples supuestos compartidos, sin los cuales no sería posible interpretar el significado de los acontecimientos. Estos contextos de mutua inteligibilidad tienden a ser imputados, por lo cual al inicio de cada interacción los interlocutores suponen que el otro comparte con él los mismos supuestos ${ }^{22}$.

¿Pero qué es lo que ocurre cuando este "conocimiento de fondo" es distinto para cada uno de los participantes en una interacción? Porque, en mayor o menor medida, esa suele ser la situación en los encuentros entre los expertos en salud y "la gente". En estos casos nos encontramos en el terreno de los malos entendidos, de lo ininteligible, lo inexplicable, lo "irracional": cada uno juzgará como "irracional" el comportamiento del otro; eso sin siquiera considerar aún el ángulo de la explicabilidad moral, que nos remitiría al complejo y dinámico mundo de las normas y valores de cada grupo social ${ }^{6,7,19,22}$.

Kleinman ${ }^{23}$, psiquiatra y antropólogo que ha estudiado los conflictos y malos entendidos que suelen suscitarse en los encuentros clínicos, parte del reconocimiento de que cada persona está guiada por determinados marcos interpretativos o modelos explicativos desde los cuales otorga sentido a sus experiencias y guía su comportamiento. Estos marcos interpretativos son los que sustentan las creencias, valores, percepciones y expectativas de cada uno de los participantes en el encuentro clínico (el paciente, su familia, el médico, otros integrantes del equipo de salud, etc.). No son necesariamente sistemáticos, ni consistentes, y en ocasiones ni siquiera inmediatamente expresables, pero las más de las veces difieren entre el médico y el paciente, lo que explica las habituales dificultades de comunicación, inconformidades y frustraciones que experimentan cada uno de ellos. Stein ${ }^{24}$ agrega un elemento más a la propuesta de Kleinman: la porción de los modelos explicativos, tanto del médico como del paciente, que permanece fuera de la conciencia de 
cada uno de ellos, y la intensa carga afectiva que ahí se origina sin que ninguno de los participantes logre percibir de dónde viene. Por lo demás, Stein ha observado que tampoco la versión científica es siempre tan objetiva y neutral como se pretendería ${ }^{24}$ : "He trabajado cercanamente con médicos que creen sinceramente que su trabajo se rige rigurosamente por el modelo explicativo científico, pero a menudo, cuando discuten la etiología de la enfermedad y el manejo del paciente especialmente cuando se sienten frustrados-, imbuyen a la enfermedad y al tratamiento con un gran número de atributos animistas y antropomorfos".

La investigación cualitativa nos ofrece valiosas herramientas para estudiar estas delicadas situaciones, averiguar qué es lo que el enfermo y su familia, por un lado, y el médico y los distintos integrantes del equipo de salud, por el otro, están pensando; qué han estado haciendo al respecto, qué es lo que piensan que clínicamente debería hacerse; cómo podría el médico explicar al paciente y sus allegados, en forma accesible y respetuosa, su propio punto de vista sobre la situación, y cómo podría escuchar el punto de vista de ellos; cómo se sienten los participantes en estas situaciones conflictivas, qué elementos emocionales se han puesto en juego para generar momentos potencialmente explosivos, y cómo manejarlos a favor de la labor terapéutica ${ }^{24}$. También los desencuentros y malos entendidos que son tan frecuentes en el terreno de las intervenciones preventivas pueden ser estudiados con provecho con los abordajes cualitativos.

\section{Cuarto nudo problemático: el sujeto despojado del significado de su propia experiencia}

Cuando leemos que Leriche (este reconocido médico y cirujano de principios del siglo XX) describía a la enfermedad como "lo que incomoda a los hombres en el ejercicio normal de su vida", y a la salud como "la vida en el silencio de los órganos" 25 , nos sentimos invitados a imaginar que quizá todavía por entonces la experiencia del sujeto merecía cierto reconocimiento por parte del médico. En nuestros días, si bien el manejo de la enfermedad fundado en los avances del conocimiento científico-técnico ha traído importantes beneficios, también ha favorecido el alejamiento del enfermo de la vivencia de su enfermedad. Al encarecer los costos de la atención médica, lo aleja de la posibilidad de recibir tales beneficios. Y al descalificar su conocimiento de sentido común, lo deja enteramente inerme frente a las contingencias que le acarrea su patología.

En lo que se refiere a la vivencia de la enfermedad, como lo señala Clavreul ${ }^{25}$, el cuerpo del enfermo ha sido enmudecido, o mejor dicho, se ha convencido al enfermo de que su cuerpo habla en un lenguaje que él no comprende, que se expresa en un idioma al cual sólo puede acceder quien conoce el código del profesional de esa ciencia ${ }^{26}$. Las enfermedades hasta hoy identificadas y nombradas por la ciencia ${ }^{27}$ se han convertido en expresiones cada vez más cifradas de ciertos modos de funcionamiento del organismo humano, que los expertos emiten desde una visión bastante lejana a la vivencia de quienes las experimentan ${ }^{16}$. La experiencia del sujeto ha venido a quedar prácticamente despojada de credibilidad. Hoy día, ya ni siquiera el silencio de los órganos puede ser considerado como una manifestación de la salud: la enfermedad puede estar ya ahí, silenciosamente presente, haciendo su mal, mucho antes de que el hombre pueda siquiera percibir la menor incomodidad en el ejercicio normal de su vida.

Distanciadas como se encuentran de su propia experiencia, las personas ya no pueden recurrir a lo que les dicen sus sentidos para saber qué es lo que ocurre con ellos mismos. Como lo señalaban Berger y Luckmann, la muy fragmentada distribución social del conocimiento nos obliga a recurrir a una compleja y confusa multitud de expertos para resolver incluso algunas de las más cotidianas situaciones, hasta el extremo en que “... no sólo me hace falta una opinión autorizada, sino también una opinión previa acerca de cuál es la opinión más autorizada" ${ }^{17}$. En nuestros días, esto es algo verdaderamente grave para el caso de la salud y la enfermedad. Porque se espera que todo integrante de la población recurra al saber profesional para saber qué es lo que le pasa y qué es lo que debe hacer. Pero los "maravillosos" beneficios de los avances científicotécnicos de la medicina no son "para todos". Las posibilidades de ser atendido están severamente restringidas, con mayor intensidad para aquellos cuya vulnerabilidad social y económica es mayor $y$, por ende, también lo es la probabilidad de que lleguen a requerir atención médica. Es este uno de los ángulos más trágicos del panorama sanitario de nuestros días.

Por lo demás, como reflexiona Berman ${ }^{18}$, cuando el propio cuerpo llega a percibirse como irreal, se deja de ser el sujeto de sí mismo; cuando el ethos de la técnica y de la administración lo invaden todo, no parece haber ya ningún lugar en donde refugiarse. 
En cuanto a la investigación cualitativa, en este punto puede abrir la mirada del estudioso de la salud al conocimiento de los distintos mar$\cos _{\text {explicativos }}^{23}$ en los que se sustentan la percepción, comprensión y expectativas de las personas frente a sus padecimientos. Puede ayudarle a percatarse de la inmensa variedad y riqueza de significados que suele tener el cuerpo y sus funciones para cada sujeto, y la amplia gama de las rutas que a lo largo de la historia los humanos han ido inventando - y siguen inventando para enfrentar y atender las enfermedades, y para lidiar con las penosas experiencias que éstas involucran ${ }^{19}$. Esta forma de aproximación al conocimiento de la realidad le permitirá, también, mirar de cerca, si se atreve, las terribles consecuencias que acarrea la injusta distribución de los recursos en las sociedades actuales, inscritas en los cuerpos de la gente. Esto último podría llegar a convertirlo en uno más de quienes pugnan por la existencia de sistemas de salud adecuados para hacer frente a las necesidades, esta vez sí, de todos los integrantes de la población.

\section{Quinto nudo problemático: el sujeto desplazado del cuidado de su salud}

Podría parecer extraña esta enunciación, en un momento en el cual el discurso preventivo ha cobrado tal relevancia. El problema reside en que las muy legítimas esperanzas sobre las posibilidades de la prevención que florecieron a mediados del siglo XX se han convertido en una suerte de coartada para que los gobiernos de muchos países eludan el compromiso de proveer servicios médicos curativos resolutivos suficientes y de calidad para responder a las enfermedades en las que se expresa la vulnerabilidad inherente al cuerpo humano, ante la exposición a las constelaciones de riesgo que imperan en las sociedades actuales ${ }^{28}$. En cambio, la preocupación se ha centrado preferentemente en el problema de los estratosféricos costos económicos que pueden llegar a alcanzar los sistemas de salud, y la propuesta preventiva se ha reducido a una postura de "culpabilización" de los individuos por sus comportamientos "poco saludables", como si el margen de acción de las personas no estuviera estrechamente acotado por los patrones de producción y consumo que prevalecen en nuestras sociedades ${ }^{29}$.

El supuesto que parece prevalecer entre los profesionales de la salud es que el principal obstáculo para obtener los comportamientos "saludables" esperados reside en la falta de "información”. Habría, pues, que "educar” a la población.
Pero cabe preguntarse si en verdad es ese el problema más importante: que "la gente" conozca o reconozca- los riesgos definidos e identificados por los profesionales de la salud. En realidad, como ya Moscovici ${ }^{30}$ lo estudiaba, muchos elementos del conocimiento experto terminan por filtrarse y permear, con diversos grados de fidelidad o distorsión, al conocimiento de sentido común; también lo muestran así los sugerentes hallazgos y reflexiones de los estudiosos de la racionalidad médica ${ }^{19}$.

En cambio, podría resultar mucho más preocupante, con esta pretensión de "cientifización del hombre común”, la manera en la cual este tipo de versión científica del mundo despoja a las personas de la confianza en su propia experiencia, las deja sin esa sensación de orientación que da el sentido común, de "saber lo que hay que hacer" frente a ciertas eventualidades de la vida cotidiana $^{17}$, las empobrece radicalmente en su capacidad de decisión, en el manejo de las situaciones en las que se encuentran involucradas, y las enajena de toda capacidad autónoma para una búsqueda propia. Si antes aludí a cómo el sujeto se encuentra casi totalmente inerme frente a su patología, ahora tendría que añadir que también lo está frente a las constelaciones de riesgos que la originan, descalificado no sólo para identificar qué es lo que le ocurre, sino también para formular alguna idea propia sobre lo que podría hacer para protegerse. Las propuestas normativas que emiten los expertos se basan en una sola y uniforme vía de solución, que se imponen ciega y autoritariamente. No se deja el menor resquicio para el surgimiento ni siquiera del germen de alguna idea potencialmente creativa por parte de quienes exprimentan en su propia carne los problemas de salud.

La investigación cualitativa nos permite observar cómo muchas de las prácticas así prescritas son de un alcance bastante limitado, y en no pocas ocasiones, resultan imposibles de realizar en las condiciones en las que vive buena parte de los habitantes de los países latinoamericanos. Las propuestas preventivas de esta naturaleza resultan, por decir lo menos, bastante poco coherentes, y en no pocas ocasiones llega a parecer casi una burla para aquellos a quienes van dirigidas.

Otra es la situación cuando se estudian con mayor cuidado y profundidad las posibilidades de fertilización mutua entre el saber "de la gente" y el saber "experto" para la construcción de vías hacia el despliegue de alternativas verdaderamente valiosas y posibles para la construcción de la salud por parte de sus protagonistas ${ }^{6,7,19}$. Nume- 
rosos ejemplos podrían encontrarse sobre las modalidades generadas a lo largo de muchas generaciones para garantizar entornos ambientales y condiciones de vida verdaderamente sustentables y radicalmente menos nosógenos que los que prevalecen hoy en día.

\section{Conclusión: hacia la recuperación del lugar del sujeto}

Quienes están convencidos de las bondades de la versión positivista de la ciencia, y comparten el valor casi absoluto que se le otorga en nuestros días a la información así producida, podrían aspirar a que la conciencia del hombre común se acercara cada vez más a esa visión desde la cual se genera el saber científico, independientemente de que exista o no para él la posibilidad de beneficiarse de ello, y sin preocuparse de que esta forma de relación con el mundo haya llevado al sujeto (tanto al hombre común como al experto) a la abdicacion de su propio lugar como generador de conocimiento, como intérprete y como protagonista de cada uno de sus movimientos.

Pero cabría preguntarnos si realmente será ese el camino hacia un mejor posicionamiento para un sujeto autoconciente, no enajenado, en el cuidado de la salud. Porque quizá, como lo señala Berman, la relación con el mundo que brota de una perspectiva que intenta conocerlo en el acto mismo de distanciarse de él, reducirlo y, en ocasiones, incluso destruirlo, favorece lo que él denomina una conciencia no participativa, la cual conduce a situaciones nada saludables, ni para el cuerpo ni para la mente: "La conciencia científica es una conciencia alienada: no hay un asociación ectásica con la naturaleza, más bien hay una total separación y distanciamiento de ella. Sujeto y objeto siempre son vistos como antagónicos. Yo no soy mis experiencias y por lo tanto no soy realmente parte del mundo que me rodea. El punto final lógico de esta visión del mundo es una sensación de reificación total: todo es un objeto ajeno, distinto y aparte de mí. Finalmente yo también soy un objeto, también soy una 'cosa' alienada en un mundo de otras cosas igualmente insignificantes y carentes de sentido. Este mundo no lo hago yo: al cosmos no le importo nada y no me siento perteneciente a él. De hecho, lo que siento es un profundo malestar en el alma"18.

Con estas reflexiones en mente, imaginemos una vez más al heterogéneo mosaico constituido por quienes forman parte de las sociedades latinoamericanas contemporáneas. Pensemos en la diversidad de perspectivas y conductas que los integrantes de los distintos grupos podrían tener frente a los servicios de salud, tanto preventivos como curativos, que les son accesibles. Éstas serán tan variadas como lo son sus ubicaciones en la sociedad y sus visiones del mundo ${ }^{19}$. Es evidente que no todos concederán la misma importancia a "lo útil" o "lo eficaz", ni todos encontrarán igualmente "benéfico" lo mismo, en tanto que no todos comparten los mismos puntos de vista, las mismas posibilidades, ni las mismas jerarquías de valores, todo lo cual tiene sus propias y complejas raíces.

Así, aún en condiciones ideales de información, lo más probable es que no se produzca la adopción de un concepto uniforme sobre cómo cuidar la salud, ni tampoco un conjunto idéntico de prácticas para enfrentar los distintos tipos de enfermedades. En esas circunstancias, ¿por qué inhibir el despliegue del amplio abanico de posibilidades y la diversidad de recursos generados por los distintos grupos de la población, a través de las búsquedas de muchas generaciones, para el cuidado y la construcción de su propia salud, en sintonía con los significados que ellos mismos les atribuyen ${ }^{23}$ ? ¿Por qué no indagar las nuevas posibilidades que podrían surgir de la conjunción de dichas prácticas con los beneficios que pudiera ofrecer el saber "experto"?

Esto requeriría, sin duda, de una mayor apertura en la mentalidad de los "expertos", para que en lugar de ignorar, descalificar, destruir o anular los recursos personales y culturales con los que cuenta "la gente", se decidieran a estudiar los beneficios que podrían venir de la conjugación de éstos con su propio saber.

En cuanto a "la gente", como hemos dicho, en su mundo el saber "experto" suele irse filtrando poco a poco hasta permear cada uno de los espacios de ese mosaico multicultural en el que vive, a su propio ritmo y con sus propios tiempos para cada caso. Al entrar en contacto algunos de los elementos del saber experto con estas particulares mentalidades y con las redes de significados tejidas en cada uno de esos contextos, todo se entremezcla para modificar las distintas certezas que cada uno tenía sobre el mundo, y producir los más diversos resultados. Todo eso está ahí, en el mundo en el que vive "la gente", para ser indagado con abordajes tan abiertos y respetuosos como los que ofrece la investigación cualitativa.

Fertilizaciones mutuas de esta naturaleza podrían potenciar el avance hacia la construcción de nuevas rutas hacia la construcción de la salud y el cuidado de la enfermedad que incluyeran ele- 
mentos tomados de esta más rica diversidad de alternativas, siempre abierta a la creatividad de los sujetos participantes, con la disposición de aventurarse en busca de nuevos y mejores destinos para nuestra especie.

$\mathrm{Al}$ devolver al sujeto el lugar que le corresponde como perceptor y productor de significados para sus propias experiencias, en el centro del escenario de la construcción de sus interpretaciones del mundo, y en particular en los cursos de acción que desa ensayar para la edificación de su salud y el cuidado de sus enfermedades, se haría posible, entre otras cosas, disminuir la enorme brecha cada día más ancha - entre quienes diseñan las políticas de salud, quienes las operan y quienes se espera que las reciban (que, sin embargo, tienden más bien a rechazarlas): "la gente", que en realidad es un conjunto de sujetos inmersos en su cultura, con los más diversos grados de capacidad imaginativa y potencialidad creadora.

Por lo demás, no habría que esperar que en este escenario imaginario los sucesos se produjeran en forma tersa y libre de conflicto. Al alzarse la voz de aquellos a quienes se ha mantenido en silencio en medio de los estragos ocasionados por la desigualdad y la injusticia social, la diversidad de posiciones encontradas no podrá dejar de expresarse. La investigación cualitativa puede auxi- liar en la identificación de los diferentes intereses en juego en cada una de las situaciones que ponen en riesgo la salud de quienes integran los distintos grupos de la población. Puede ser también un instrumento de gran valor para sumergirse en la complejidad de los desencuentros suscitados por los distintos mundos en los que viven "los expertos" y "la gente"31, con toda la diversidad de marcos explicativos en los que se sustentan la percepción, comprensión, cursos de acción y expectativas de las personas.

Mucho más complicado será lidiar con los irreconciliables intereses políticos y económicos en juego. Los gobiernos que han abandonado el concepto de la salud como un bien público, y que eluden cada día más la responsabilidad de atender los perfiles de daños a la salud de sus poblaciones, no serán proclives a aceptar enfoques de esta naturaleza. De ahí que el investigador que decida incursionar por los caminos de esta modalidad de aproximación -la investigación cualitativa, en especial si su enfoque es crítico- no pueda ignorar que se interna en terrenos políticamente espinosos. Pero no debe olvidar, tampoco, la fortaleza epistemológica, que aquí va íntimamente vinculada a la ética -e incluso a la estética-, de los fundamentos que respaldan a este tipo de quehacer ${ }^{4,6,7,8}$. 


\section{Referencias}

1. Schütz A. El problema de la realidad social. Buenos Aires: Amorrortu; 1995.

2. Morin E. La inteligencia ciega. En: Morin E, editor Introducción al pensamiento complejo. Barcelona: Gedisa; 1997. p. 27-35.

3. Habermas J. Conocimiento e interés. Madrid: Taurus; 1968.

4. Kincheloe J, McLaren P. Rethinking critical theory and qualitative research. En: Denzin N, Lincoln Y, editors. The Sage handbook of qualitative research. $3^{\text {th }}$ ed. Thousand Oaks, CA: Sage Publications; 2005. p. 303-342.

5. Martínez C, Leal G. Cuando nos hablan de salud ¿podemos confiar en los expertos? El Cotidiano 2000; 103:73-81.

6. Guerriero ICZ Dallari SG. A necessidade de diretrizes éticas adequadas às pesquisas qualitativas em saúde. Cien Saude Colet 2008; 13(2):303-311.

7. Bosi MLM. Pesquisa qualitativa em saúde coletiva: panorama e desafios. Cien Saude Colet 2012; 17(3): 575-586.

8. Minayo MCS. Los conceptos estructurantes de la investigación cualitativa. Salud Colectiva 2010; 6(3):251-261.

9. Denzin N, Lincoln Y, editors. The Sage handbook of qualitative research. $3^{\text {th }}$ ed. Thousand Oaks, CA: Sage Publications; 2005.

10. Córdova A, Leal G, Martínez C. Críticas sobre la reducción positivista de la corporeidad. En: PérezRincón $\mathrm{H}$, editor. Imágenes del cuerpo. México: Cuadernos de la Gaceta Fondo de Cultura Económica; 1992. p. 107-128.

11. Martínez C. La familia y la estructuración psíquica del sujeto. México: Académicos de CBS, UAM-X; 2007.

12. McDougall J. Teatros del cuerpo. Madrid: Julián Yébenes; 1995.

13. Winnicott D. La mente y su relación con el psiquesoma. En: Winnicott D. Escritos de pediatría y psicoanálisis. Barcelona: Paidós; 1999. p. 325-340.

14. Minayo MCS. Análise qualitativa: teoria, passos e fidedignidade. Cien Saude Colet 2012; 17(3):621-626.

15. Martínez C, Chapela MC, Ruiz-Velasco V, coordenadores. En el juego de los espejos. Multi, inter, transdisciplina e investigación cualitativa en salud. México: Académicos de CBS, UAM-X; 2013.

16. Martínez C. Los múltiples significados de la salud. Un recorrido bajo la guía de Canguilhem. En: Martínez C, editor. Seis Miradas sobre la salud y su relación con el mundo social. México: Académicos de CBS, UAM-X; 2008

17. Berger P, Luckmann TH. La construcción social de la realidad. Buenos Aires: Amorrortu; 1995.

18. Berman M. El reencantamiento del mundo. Santiago de Chile: Cuatro Vientos; 1995.
19. Nascimento MC, Barros NF, Nogueira MI, Luz MT. A categoria racionalidade médica e uma nova epistemologia em saúde. Cien Saude Colet 2013; 18(12): 3595-3604.

20. Van den Hoonaard W. Re-imaginando o "sujeito": considerações éticas e conceituais sobre o participante de pesquisa qualitativa. Cien Saude Colet 2008; 13(2):371-379.

21. Garfinkel H. Studies in ethnomethodology. Cambridge G.B.: Polity Press; 1984.

22. Heritage J. Etnometodología. En: Giddens A, Turner J. La teoría social hoy. México: Alianza Editorial CONACULTA; 1991. p. 290-350.

23. Kleinman A. Patients and healers in the context of culture. Berkeley: University of California Press; 1980.

24. Stein H. The psychodynamics of medical practice. Unconscious factors in patient Care. Berkeley: University of California Press; 1985.

25. Clavreul J. El orden médico. Barcelona: Argot; 1983.

26. Córdova A, Leal G, Martínez C. Cabos sueltos de epistemología médica, Reporte de investigación 63. México: UAM-X, División de Ciencias Biológicas y de la Salud; 1991.

27. Organización Panamericana de la Salud (OPS). Clasificación Estadística Internacional de Enfermedades y Problemas Relacionados con la Salud. V. 1, 2, 3. Décima Revisión. Washington: OPS; 2008.

28. Martínez C, Leal G. La prevención y la cura. En busca de nuevos equilibrios. En: Creatividad y quehacer científico en la UAM-Xochimilco 2001. México: UAM-X; 2002. p. 523-534.

29. Martínez C, Leal G. Epidemiological transition model or illusion? A look at the problem of health in Mexico. Soc Sci Med 2003; 3(57):539-550.

30. Moscovici S. La psychanalyse son image et son public. Vendôme: Presses Universitaires de France; 1961.

31. Martínez C. Cultivando la relación médico-paciente en escenarios difíciles. En: Martínez C, editor. Por los caminos de la investigación cualitativa. Exploraciones narrativas y reflexiones en el ámbito de la salud. México: UAM-X; 2010. p. 41-59.

Artigo apresentado em 20/06/2013

Aprovado em 05/08/2013

Versão final apresentada em 17/08/2013 